\title{
A singular value decomposition based generalized finite difference method for fluid solid interaction problems
}

\author{
P. Yu, K. S. Yeo, X. Y. Wang \& S. J. Ang \\ Department of Mechanical Engineering, \\ National University of Singapore, 9 Engineering Drive 1, \\ Singapore 117576, Singapore
}

\begin{abstract}
A hybrid meshfree-Cartesian grid method is proposed for simulating three dimensional fluid-solid interaction (FSI) problems involving rigid bodies with large boundary motions. The rigid body is embedded and enveloped by a cloud of mesh-free nodes, which convect with the motion of the body against a background of Cartesian nodes. Spatial discretization is accomplished by the combination of a Generalized Finite Difference (GFD) method and conventional finite difference (FD) method applied to the meshfree and Cartesian nodes respectively. Error minimization in GFD is carried out by singular value decomposition (SVD). A time-implicit iterative procedure is employed to compute the new/evolving position of the immersed bodies together with the dynamically coupled solutions of the flow field and bodies. The present method is applied to simulate the FSI problems of freely falling bodies in quiescent flow and freely rotating bodies in shear flow. The good agreement with published results validates the ability of the present hybrid meshfree-Cartesian grid scheme for solving FSI problems in 3D.
\end{abstract}

Keywords: fluid-solid interaction, generalized finite difference method, singular value decomposition, projection method.

\section{Introduction}

Many flows of practical interest in engineering and bioengineering involve fluidsolid interaction (FSI). Typical examples include the vibration of bridges and buildings to winds, the flutter of aircraft wings, the flow of blood through 
arteries, and the swimming and flying of animals or insects. The challenge remains for the numerical simulation of this class of flow problems because the position, and in certain situations the shape, of the interface is not known a priori and has to be determined by dynamically coupling the motion of the body and the flow of the surrounding fluid.

From the point of view of methods for moving boundary treatment, the numerical methods for the FSI problems in literature may be divided into two categories: the arbitrary Lagrangian-Eulerian (ALE) or moving grid approach and the fixed or Cartesian grid approach. In the ALE approach, the governing equations for the fluid flow are adapted and solved on a grid which deforms/moves with the interface of the body [1]. An ALE approach associated with finite element method (FEM) has been developed by $\mathrm{Hu}$ and his co-workers $[2,3]$ and recently been improved by Choi [4] and Hu et al. [5]. The method has been applied to simulate the motion of a large number of particles in fluids [3-5]. An ALE formulation based on finite volume method (FVM) has been proposed by Papadakis [6]. The method has been used to investigate the wave propagation in a flexible tube and good agreement has been found between his results and the existing analytic solutions or numerical results. Although the ALE based approaches are the most widely used techniques so far, the limitation of constantly regenerating the mesh to accommodate the changing solution domain, which inevitably results in increased costs in mesh administration and data interpolation, and a possible increase in numerical errors, hampers its development.

The immersed boundary method (IBM) [7] is one of the most prominent and earliest techniques based on the fixed grid approach. It has the simplicity of grid system and corresponding efficiency in computation. The main drawback of the IBM is that the piecewise continuous solution across the immersed boundary tends to be smeared by the distribution of the singular forces over several grid nodes, leading to reduced spatial resolution and accuracy near the boundary (frequently of first order). A number of improved IBMs have been proposed, and only those applied to FSI problems will be mentioned here. Tai et al. [8] have developed an immersed object method for 3D FSI flow and applied it to simulate 3D unsteady blood flow and blood-leaflets interaction in a mechanical heart valve. Borazjani et al. [9] has proposed a sharp-interface curvilinear immersed boundary method to simulate FSI problems involving $3 \mathrm{D}$ rigid bodies undergoing large displacements. Gilmanov and Acharya [10] have developed a hybrid immersed boundary and material point method for FSI problems involving 3D deformable bodies.

In the present study, a numerical method is proposed for the FSI problems of non-deformable rigid body based on a hybrid meshfree-Cartesian grid. The body motion is governed by Newton's second law. The force and torque acting on the bodies are obtained by integrating the fluid traction force over the surface of the rigid body. Meshfree nodes are employed to discretize complex immersed boundaries. The boundary nodes, together with the cloud of meshfree nodes that cluster around the immersed bodies, which can convect against a background of Cartesian nodes, are used to track the motion of the bodies. Spatial discretization 
is accomplished by the combination of a Generalized Finite Difference (GFD) method and conventional finite difference method (FDM) applied to the meshfree and Cartesian nodes respectively. The mixed Lagrangian-Eulerian form of the Navier-Stokes equations on convecting nodes is integrated by a secondorder Crank-Nicolson based projection method. Error minimization in GFD is carried out by singular value decomposition (SVD). A time-implicit iterative procedure is employed to compute the new/evolving position of the immersed bodies together with the dynamically coupled solutions of the flow field and body.

\section{Method}

In the present study, the motion of the body is described by convecting the meshfree nodes against a background of Cartesian nodes. The flows of the incompressible and viscous fluid on Cartesian nodes and on the convecting mesh-free nodes are governed by Navier-Stokes equations in the standard form and a mixed Lagrangian-Eulerian form $[1,11]$, respectively. The standard 7 point central finite difference scheme is applied at all Cartesian nodes that do not have meshfree node(s) within its closed $[-\Delta x, \Delta x] \times[-\Delta y, \Delta y] \times[-\Delta z, \Delta z]$ neighbourhood. Cartesian nodes that are overlapped by a meshfree nodal cloud are excluded from flow computation to reduce unnecessary computations as well as to maintain good nodal quality. The meshfree nodes that are located on and around the boundary of the immersed bodies, and the Cartesian nodes that have one or more of cloud nodes within their $[-\Delta x, \Delta x] \times[-\Delta y, \Delta y] \times[-\Delta z, \Delta z]$ neighbourhoods are subject to the SVD-based generalized finite difference (GFD) treatment $[12,13]$. A second-order implicit projection method, based on a fractional-step Crank-Nicolson scheme is applied for the temporal treatment of the flow equations. The detailed information on the numerical treatment can be found in the previous works of Chew et al. [11], Ang et al. [12], and Wang et al. [13]. Here the attention is focused on the dynamics of rigid body and the coupling of its motion to the flow of the surrounding fluid.

The configuration of the rigid body is specified geometrically in a separate frame, termed the body frame. The body frame will translate and rotate with respect to the global/computational frame in accordance with the dynamic motion of the body. For simplicity, the origin of the body frame is placed at the centre of mass of the body. If we use a vector $X_{c}(t)$ to describe the translation of the centre of mass of the body and a $3 \times 3$ matrix $R_{c}(t)$ to describe the rotation/orientation of the body about the centre of mass, the transformation of an arbitrary point $X_{P O}(0)$ on the rigid body in the body frame into a new position $X_{P 0}(t)$ in the global frame can be expressed as:

$$
X_{P 0}(t)=X_{c}(t)+R_{c}(t) X_{P 0}(0)
$$

The motion of the rigid body obeys Newton's laws. The linear and angular momentum equations have the forms given by:

$$
P(t)=M v(t)
$$




$$
L(t)=I(t) \omega(t)
$$

where the $P(t)$ and $L(t)$ are the total linear and angular momentums of the rigid body, respectively, $v(t)$ and $\omega(t)$ are the linear and angular velocity of the rigid body, respectively, $M$ is the mass of the rigid body and $I(t)$ is the inertia tensor of the rigid body. The relationship between $R_{c}(t)$ and $\omega(t)$ is linked by:

$$
\dot{R}_{c}(t)=\omega(t) \times R_{c}(t)=\left|\begin{array}{ccc}
0 & -\omega_{3} & \omega_{2} \\
\omega_{3} & 0 & -\omega_{1} \\
-\omega_{2} & \omega_{1} & 0
\end{array}\right|\left|\begin{array}{l}
R_{c 1} \\
R_{c 2} \\
R_{c 3}
\end{array}\right|
$$

The relationships between the total force $F(t)$ on the rigid body and the total linear momentum $P(t)$ is:

$$
\dot{P}(t)=F(t)
$$

Similarly, the relationship between the total torque $\tau(t)$ on the rigid body and the total angular momentum $L(t)$ is:

$$
\dot{L}(t)=\tau(t)
$$

To update the position and orientation of the body, a Crank-Nicholson liked method is used to integrate the above dynamic equations.

A time-implicit iterative procedure is applied for the coupling between the solid and fluid solver. We assume that the solutions of the flow field and body position and motion have been obtained at the time step $n$. Then we guess the solution of body motion at the new time step $n+1$. After that, the flow field is computed based on the new position and motion of the body. The total body force $F(t)$ and torque $\tau(t)$ are then updated and so do the position and motion of the body. A number of these sub-iteration steps are carried out at each time step until the coupled fluid/structure solution converges adequately.

\section{Results and discussion}

\subsection{Free falling of a sphere in an infinite medium}

The motion of immersed body in fluids has attracted much attention due to its academic value and the related engineering significance associated with sedimentation procedure, fluidised bed etc. Here we will consider the motion of a sphere under the action of gravity in an infinite domain filled with a Newtonian fluid of viscosity $\mu$ and density $\rho_{f}$. The computational domain is presented schematically in Figure 1, which has the boundaries of $X= \pm L, Y= \pm W, Z= \pm H$. A rigid sphere of diameter $D$ and density $\rho_{s}$ is initially placed at $(0,0, H / 2)$. To minimize the effect of the boundaries, the lengths of the computational domain are set as $L=7.5 D, W=7.5 D, H=12.5 D$ and the Neumann boundary condition is applied at all the boundaries. 
Fluid Structure Interaction V 29

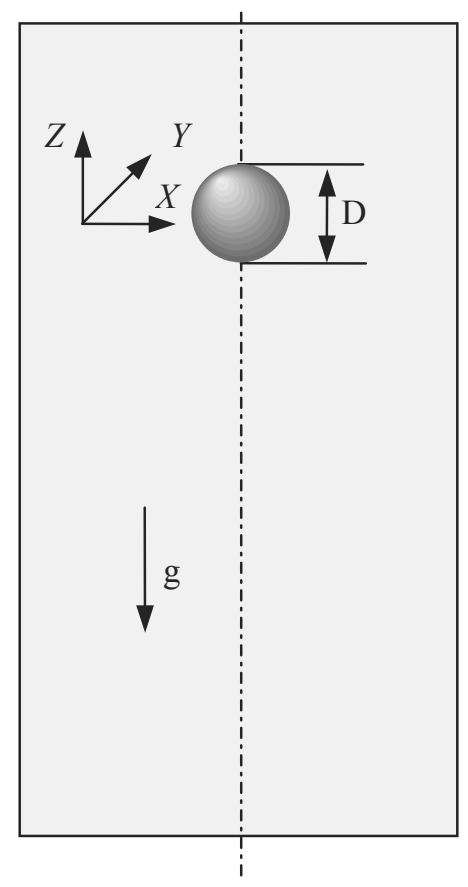

Figure 1: Computational domain of the flow induced by a freely falling sphere.

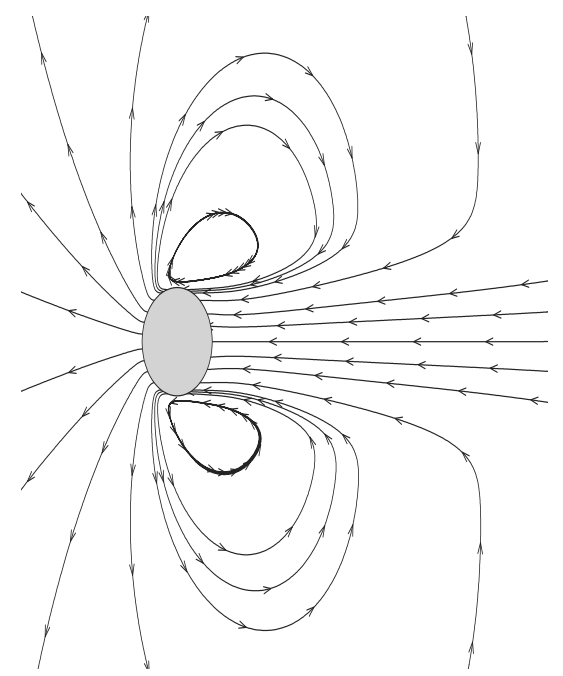

Figure 2: Streamtraces of flow field around a freely falling sphere at $\mathrm{Re}=$ 100; the sphere has reached its terminal velocity. 
The Reynolds number of the falling sphere was based on its diameter and the terminal velocity $u_{T}$ in an infinite medium, which can be written as:

$$
\operatorname{Re}=\rho_{f} D u_{T} / \mu
$$

A relationship for the drag coefficient introduced by Abraham [14] was used to determine the terminal velocity:

$$
C_{d}=\frac{24}{9.06^{2}}\left(\frac{9.06}{\sqrt{\mathrm{Re}}}+1\right)^{2}
$$

The detailed fluid parameters used in the present simulation are: $D=1 \mathrm{~m}, \rho_{s}=$ $1.05 \mathrm{~kg} / \mathrm{m}^{3}$ and $\rho_{f}=1.05 \mathrm{~kg} / \mathrm{m} 3$. The viscosity $\mu_{\mathrm{f}}$ of the fluid is changed to obtain different Reynolds number.

Figure 2 presents the streamtraces of the flow field in the $x-z$ central planes at $\mathrm{Re}=100$. Note that the present streamtraces is plotted against the global frame. If the reference frame is attached to the sphere, a pair of standing eddies behind the sphere, with its length consistent with that of a uniform flow past a stationary sphere at the same Re, can be observed.

The terminal velocity $u_{T}$ of the freely falling sphere at different $\operatorname{Re}$ is presented in Figure 3. The solid line is computed based on the equilibrium of external forces acting on the sphere; comprising the weight, the buoyancy force and the hydrodynamic drag given by Eq. (8). The empty square symbols represent the results obtained by the present simulations. The two results show very good agreement.

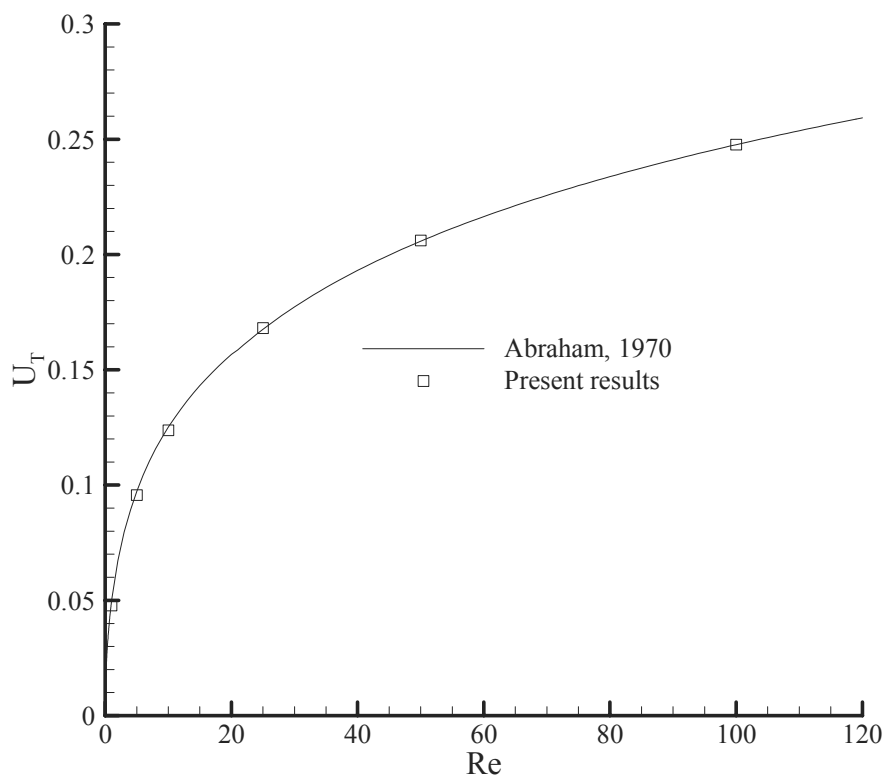

Figure 3: Comparison of terminal velocity at different Re. 


\subsection{A freely rotating sphere in simple shear flow}

The present case considers a freely rotating sphere in simple shear flow at finite Reynolds number. The densities of the sphere and the fluid are identical so that the sphere can freely suspend in the fluid. The shear flow Reynolds number is defined as $\operatorname{Re}=G R^{2} / v$, where $G$ is the shear rate of the Cartesian simple shear flow $\vec{U}=(-G Y, 0,0), R$ is the radius of the sphere, and $v$ is the kinematic viscosity of the fluid. The sphere is located at the centre of the channel with boundaries at $X= \pm L, Y= \pm H, Z= \pm W$. The size of domain is limited at $L=2 R$, $H=2 R$ and $W=20 R$ to maintains the channel Re at values which were found to remain stable. At the side walls, a condition of return to simple shear flow, $\vec{U}=-G Y \vec{e}_{x}$, is imposed. While at the inlet and outlet $(X= \pm L)$, the Neumann boundary condition is applied.

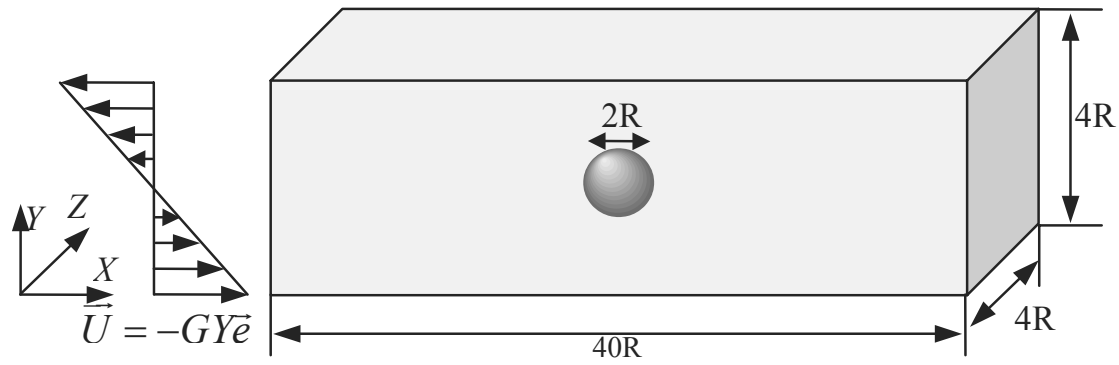

Figure 4: Schematic of computational domain of a neutrally buoyant sphere in shear flow.

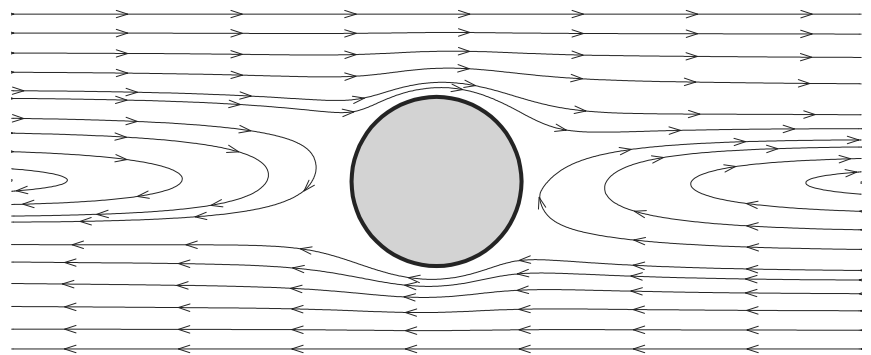

Figure 5: $\quad$ Flow field around a freely rotating sphere in simple shear flow at $\operatorname{Re}=20$.

Figure 5 shows the streamtraces of the flow field in $x-y$ central plane at $\mathrm{Re}=$ 20. Due to the presence and the rotation of the sphere, there are reverse flows on both sides of the sphere. Also, the flows on the two sides are not symmetric due to the rotation of the sphere. The normalized rotation rate of the sphere (scaled 
by the shear rate $G$ ) is computed and compared with the data in literature as shown in Figure 6. The rotation rate increases with a decrease in $R e$ and approaches the value of 0.5 when $R e \rightarrow 0$, the Stokes limit. The present results agree well with those of Mikulencak and Morris [15]. The small discrepancy may be due to the difference domain size and boundary conditions used.

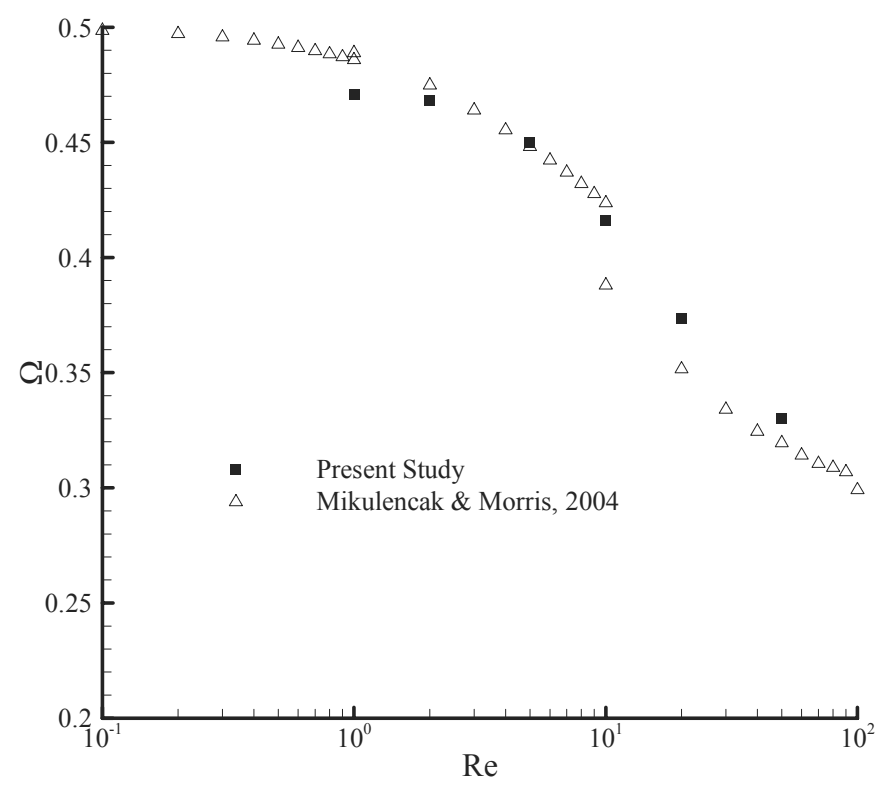

Figure 6: Rotation rates for a freely suspended sphere in simple shear flow.

\section{Conclusion}

In the present paper, we extend the SVD-GFD method based on the hybrid meshfree-Cartesian grid [11-13] to simulate 3D FSI problems for rigid bodies undergoing large boundary motion. The boundary geometry of the rigid body is precisely represented by the meshfree nodes, which convect with the motion of the body. A mixed Lagrangian-Eulerian form of the incompressible NavierStokes equations governs the flow at meshfree nodes. Discretization at meshfree nodes is performed with a singular value decomposition based generalized finite difference (SVD-GFD) scheme. Discretization at Cartesian nodes in the bulk of the computational domain is carried out by cost-efficient accurate standard finite difference method. A fraction-step projection method is used to integrate the flow equations. The present scheme is validated by two FSI problems: a freely falling sphere in an infinite fluid medium for translational motion and a freely rotating sphere in a simple shear flow for rotational motion. The good agreement of the present numerical results with the published results demonstrates the effectiveness and potential of the present hybrid meshfree-Cartesian grid scheme for solving complex FSI problems in 3D. 


\section{Acknowledgement}

This work was supported by TSDI Grant TDSI / 07-001 / 1A.

\section{References}

[1] Hirt, C.W., Amsden, A.A. \& Cook, J.L., An arbitrary Lagrangian-Eulerian computing method for all flow speeds. Journal of Computational Physics, 14, pp. 227-253, 1974.

[2] Hu, H.H., Joseph, D.D. \& Crochet, M.J., Direct simulation of fluid particle motion. Theoretical and Computational Fluid Dynamics, 3, pp. 285-306, 1992.

[3] Hu, H.H., Direct simulation of flows of solid-liquid mixtures. International Journal of Multiphase Flow, 22, pp. 335-352, 1996.

[4] Choi, H.G., Splitting method for the combined formulation of the fluidparticle problem. Computer Methods in Applied Mechanics and Engineering, 190, pp. 1367-1378, 2000.

[5] Hu, H.H., Patankar N.A. \& Zhu, M.Y., Direct numerical simulations of fluid-solid systems using the arbitrary Lagrangian-Eulerian technique. Journal of Computational Physics, 169, pp. 427-462, 2001.

[6] Papadakis, G., A novel pressure-velocity formulation and solution method for fluid-structure interaction problems. Journal of Computational Physics, 227, pp. 3383-3404, 2008.

[7] Peskin, C.S., Numerical analysis of blood flow in the heart. Journal of Computational Physics, 25, pp. 220-252, 1977.

[8] Tai, C.H., Liew, K.M., \& Zhao, Y., Numerical simulation of 3D fluidstructure interaction flow using an immersed object method with overlapping grids. Computers and Structures, 85, pp. 749-762, 2007.

[9] Borazjani, I., Ge, L. \& Sotiropoulos, F., Curvilinear immersed boundary method for simulating fluid structure interaction with complex 3D rigid bodies. Journal of Computational Physics, 227, pp. 7587-7620, 2008.

[10] Gilmanov, A. \& Acharya, S., A hybrid immersed boundary and material point method for simulating 3D FSI problems. International Journal for Numerical Methods in Fluids, 56, pp. 2151-2177, 2008

[11] Chew, C.S., Yeo, K.S. \& Shu, C., A generalized finite-difference (GFD) ALE scheme for incompressible flows around moving solid bodies on hybrid meshfree - Cartesian grids. Journal of Computational Physics, 218, pp. 510-548, 2006.

[12] Ang, S.J., Yeo, K.S., Chew, C.S. \& Shu, C., A singular-value decomposition (SVD)-based generalized finite difference (GFD) for closeinteraction moving boundary flow problems. International Journal for Numerical Methods in Engineering, 76, pp. 1892-1929, 2008.

[13] Wang, X.Y., Yeo, K.S., Chew, C.S. \& Khoo, B.C., A SVD-GFD scheme for computing 3D incompressible viscous fluid flows. Computers \& Fluids, 37, pp. 733-746, 2008. 
34 Fluid Structure Interaction V

[14] Abraham, F., Functional dependence of drag coefficient of a sphere on Reynolds number. Physics of Fluids, 13, pp. 2194-2195, 1970.

[15] Mikulencak, D.R. \& Morris, J.F., Stationary shear flow around fixed and free bodies at finite Reynolds number. Journal of Fluid Mechanics, 520, pp. 215-242, 2004. 